PAPER • OPEN ACCESS

Poly(ester amide) microspheres are efficient vehicles for long-term intracerebral growth factor delivery and improve functional recovery after stroke

To cite this article: Tamar Memanishvili et al 2020 Biomed. Mater. 15065020

View the article online for updates and enhancements.

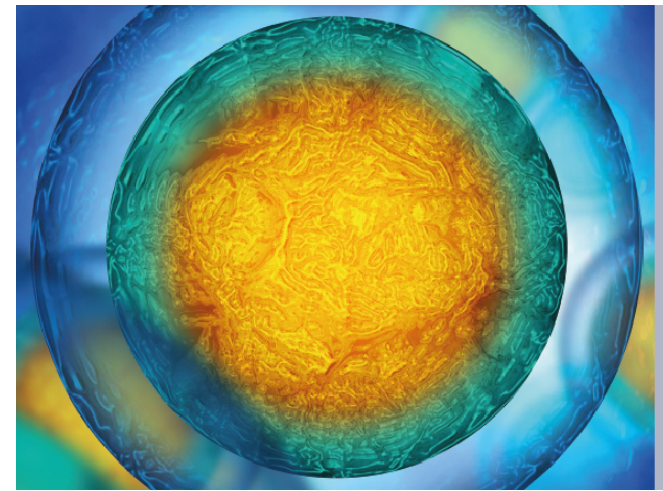

Biophysical Society $I O P \mid$ ebooks $^{\text {fw }}$

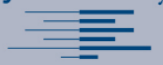

Your publishing choice in all areas of biophysics

research.

Start exploring the collection-download the first chapter of every title for free. 


\title{
Biomedical Materials
}

\section{PAPER}

CrossMark

OPEN ACCESS

RECEIVED

9 January 2020

REVISED

15 June 2020

ACCEPTED FOR PUBLICATION

10 July 2020

PUBLISHED

20 November 2020

Original content from

this work may be used

under the terms of the

Creative Commons

Attribution 4.0 licence.

Any further distribution of this work must

maintain attribution to

the author(s) and the title

of the work, journal

citation and DOI.

\section{Poly(ester amide) microspheres are efficient vehicles for long-term intracerebral growth factor delivery and improve functional recovery after stroke}

\author{
Tamar Memanishvili ${ }^{1}$, Emanuela Monni ${ }^{1}$, Jemal Tatarishivili ${ }^{1}$, Olle Lindvall ${ }^{1}$, Alexander Tsiskaridze ${ }^{2}$, \\ Zaal Kokaia ${ }^{1,3}$ (1) and Daniel Tornero ${ }^{1,3,4}$ \\ 1 Laboratory of Stem Cells and Restorative Neurology, Lund Stem Cell Center, Lund University, Lund, Sweden \\ 2 Department of Neurology, Iv. Javakhishvili Tbilisi State University, Tbilisi, Georgia \\ E-mail: zaal.kokaia@med.lu.se and tamunamema@gmail.com
}

Keywords: microspheres, poly(ester amide), VEGF, stroke, inflammation, angiogenesis

Supplementary material for this article is available online

\begin{abstract}
Growth factors promote plasticity in injured brain and improve impaired functions. For clinical application, efficient approaches for growth factor delivery into the brain are necessary. Poly(ester amide) (PEA)-derived microspheres (MS) could serve as vehicles due to their thermal and mechanical properties, biocompatibility and biodegradability. Vascular endothelial growth factor (VEGF) exerts both vascular and neuronal actions, making it suitable to stimulate post-stroke recovery. Here, PEA (composed of adipic acid, L-phenyl-alanine and 1,4-butanediol) MS were loaded with VEGF and injected intracerebrally in mice subjected to cortical stroke. Loaded MS provided sustained release of VEGF in vitro and, after injection, biologically active VEGF was released long-term, as evidenced by high VEGF immunoreactivity, increased VEGF tissue levels, and higher vessel density and more NG2 + cells in injured hemisphere of animals with VEGF-loaded as compared to non-loaded MS. Loaded MS gave rise to more rapid recovery of neurological score. Both loaded and non-loaded MS induced improvement in neurological score and adhesive removal test, probably due to anti-inflammatory action. In summary, grafted PEA MS can act as efficient vehicles, with anti-inflammatory action, for long-term delivery of growth factors into injured brain. Our data suggest PEA MS as a new tool for neurorestorative approaches with therapeutic potential.
\end{abstract}

\section{Introduction}

Ischemic stroke causes brain damage with loss of neurons, glial cells, and vasculature, leading to longterm disability and death. Thrombolysis and thrombectomy reduce injury and functional impairments if administered during the first hours after the insult (Hacke et al 2008), but effective treatments to promote recovery in the chronic phase are lacking (Di Carlo 2009, Feigin et al 2014). Growth factors can promote neurogenesis and angiogenesis (Lee and Son 2009), two processes that play important roles for

\footnotetext{
ZK and DT have shared senior authorship

Current address: Faculty of Medicine, Department of Biomedical Sciences, Institute of Neurosciences, University of Barcelona, Barcelona, Spain.
}

functional restoration after stroke. Among growth factors, vascular endothelial growth factor (VEGF) has been shown to regulate formation of new blood vessels and proliferation and differentiation of neuronal precursors (Rosenstein et al 1998, Palmer et al 2000, Krum et al 2002, Sun et al 2003), to promote post-stroke recovery (Hayashi et al 1998, Zhang et al 2000b, Wittko et al 2009) and also to be neuroprotective (Sun et al 2003).

These findings have raised the possibility that administration of growth factors to the injured brain might be developed into a new therapeutic strategy in stroke patients. However, growth factors are large molecules with limited ability to penetrate the bloodbrain barrier (BBB) (Lo et al 2001, Pardridge 2005). Sustainable delivery of VEGF is also difficult due to high degradation rate and short half-life following 
systemic administration (Storkebaum et al 2004, Orive et al 2009).

One strategy to overcome the problems with growth factor delivery is to use biomaterials as carriers. Implantation of such materials promotes controlled and sustainable release in the microenvironment (Orive et al 2009, Elliott Donaghue et al 2014), and allows for efficient long-term delivery of growth factors into the brain parenchyma (Ferreira et al 2007, Emerich et al 2010). Polymeric microparticles appear particularly promising for this purpose (Zhuang et al 2017), and can be delivered directly into the target loaded with different drugs or molecules. For example, poly(lactic-co-glycolic-acid) (PLGA) microspheres (MS) implanted into striatum of dopamine-denervated rats provided continuous release of VEGF and glial cell line-derived neurotrophic factor (GDNF), promoting functional recovery in this model of Parkinson's disease (Herran et al 2013b). Similarly, VEGF-loaded PLGA nanospheres placed directly into the cerebral cortex improved behavioral deficits and stimulated angiogenesis in a mouse model of Alzheimer's disease (Herran et al 2013a). Supporting the usefulness of this strategy for VEGF delivery also in stroke, prolonged release of VEGF from PLGA microcarriers increased angiogenesis around the striatal implantation site and facilitated migration of immature neurons towards the ischemic tissue (Quittet et al 2015). Moreover, VEGFloaded PLGA MS with attached human neural stem cells promoted neovascularization in the stroke cavity (Bible et al 2012).

Although PLGA is the most common biodegradable polymer used for tissue engineering (Makadia and Siegel 2011), some limitations have been reported, including moderate toxic effects (Sundback et al 2005, Dailey et al 2006). Additionally, PLGA is a polyester that degrades by bulk erosion through hydrolysis of ester bonds, leading to rapid mass loss. Acidic degradation byproducts lower local $\mathrm{pH}$, which may cause cell and tissue damage (Hemmrich et al 2008, London et al 2011, Makadia and Siegel 2011, Liu et al 2012). Unlike polyesters including PLGA, after biodegradation, poly(ester amide)s (PEAs) release weaker acidic products in lower quantities, such as neutral (zwitterionic) $\alpha$-amino acids and diols, with relatively weak fatty diacids, making them less harmful for cells (Fonseca et al 2014). The PEAs belong to a less explored family of biodegradable polymers composed of naturally occurring $\alpha$-amino acids, which are released upon biodegradation promoting regenerative processes in tissues (Chu 2012, Andres-Guerrero et al 2015, Katsarava et al 2016). The PEAs have been shown to be useful for different biomedical applications (Lee et al 2002, Markoishvili et al 2002, Jikia et al 2005, Huang et al 2006, 2009, Knight et al 2014, DeFife et al Andres-Guerrero et al 2015). We were the first to demonstrate that PEA MS can provide sustained release of Wnt3A, BMP4 and cyclopamine, promoting in vitro differentiation of human induced pluripotent stem cells to cortical neurons (Memanishvili et al 2016).

Here we show that MS made of biocompatible and biodegradable PEA (composed of adipic acid, Lphenyl-alanine and 1,4-butanediol (PEA 4F4)) give rise to sustained release of loaded VEGF in vitro and, when injected into stroke-injured cerebral cortex, that both non-loaded and VEGF-loaded MS promote post-stroke recovery, the loaded ones inducing more rapid improvement. Our findings strongly support the usefulness of PEA MS for local, long-term intracerebral delivery of compounds which do not penetrate BBB. In addition, the data indicate that not only the released VEGF but also the injected PEA MS themselves promote behavioral recovery after stroke.

\section{Materials and methods}

\subsection{Generation of MS}

Poly(ester amide) 4F4, composed of adipic acid, Lphenyl-alanine and 1,4-butanediol (4F4; referring to number of methylene groups in adipic acid, phenylalanine and number of methylene groups in butanediol, respectively) was synthesized by solution polycondensation of di-p-toluenesulfonic acid salt of bis $(\alpha$-amino acid) $\alpha, \omega$-alkylene diesters with active diesters of dicarboxylic acid as previously described (Tsitlanadze et al 2004). Polymer structure is as follows:

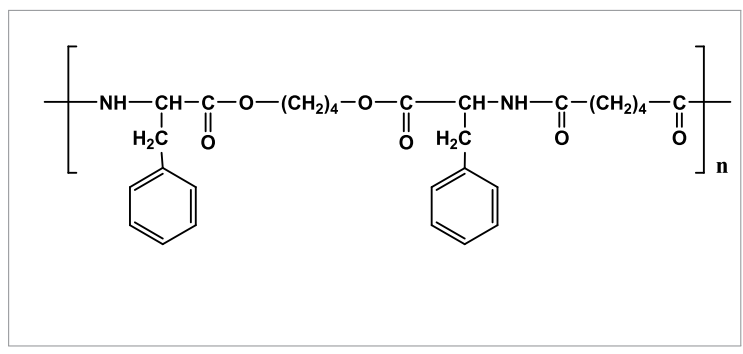

We explored the possibility to load PEA 4F4 MS with VEGF for sustainable release. We used VEGF 121, which lacks heparin-binding domains and, therefore, is more diffusible than other isoforms (Kaplan et al 2016). Also, VEGF 121 is more angiogenic (Zhang et al 2000a). The growth factor was loaded in MS using water-in-oil-in-water (W1/O/W2) double emulsion solvent evaporation technique, which we have previously used for successfully loading PEA 4F4 MS with Wnt3A, BMP4 and cyclopamine (Memanishvili et al 2016). Briefly, $250 \mathrm{mg}$ of PEA 4F4 were dissolved in $5 \mathrm{ml}$ chloroform $(\geqslant 99.5 \%, 100-200 \mathrm{ppm}$ amylenes, Sigma-Aldrich) at room temperature and $2.5 \mu \mathrm{g}$ of lipase (from porcine pancreas Type II, 100-500 units $\mathrm{mg}^{-1}$, SigmaAldrich) were added. Lyophilized VEGF 121 (Pepro 
Tech) was resuspended according to the manufacturer's instructions to obtain a stock solution of protein. To prepare the primary emulsion, $300 \mu \mathrm{l}$ of protein solution containing $30 \mu \mathrm{g}$ recombinant human VEGF 121 in $0.1 \%$ bovine serum albumin (BSA) in milli-Q water (Merck) were emulsified with polymer solution using sonicator (Branson Sonifier SLPe150D) with a microtip on ice for $2 \mathrm{~min}$. This primary emulsion was then emulsified with $10 \mathrm{ml}$ pre-filtered $2.5 \%(\mathrm{w} / \mathrm{v})$ polyvinyl alcohol (PVA, 13-23 $000 \mathrm{Mw}$ $87 \%-89 \%$ hydrolyzed, Sigma-Aldrich) solution. The resulting double emulsion was immediately transferred into a beaker containing $800 \mathrm{ml}$ of the prefiltered $0.5 \%(\mathrm{w} / \mathrm{v})$ PVA solution and stirred continuously (IKA magnetic stirrer, medium speed) for $24 \mathrm{~h}$ at room temperature to allow the organic solvent to evaporate.

Following complete evaporation, solidified PEA 4F4 MS were washed three times with milli-Q water and harvested via vacuum filtration. The particle suspension was passed through a $40 \mu \mathrm{m}$ pore sterile filter (BD Biosciences) and freeze-dried (FreeZone Plus 6, Labconco freeze dry system) for $48 \mathrm{~h}$. Dried PEA 4F4 MS were stored at $-20{ }^{\circ} \mathrm{C}$ to prevent inactivation of VEGF. As control, non-loaded PEA 4F4 MS were prepared using the same method as described above but without protein. For sterilization, PEA 4F4 MS were placed on a bench-top/orbital 3D shaker (VWR) with speed at $20 \mathrm{rpm}$ and exposed to ultraviolet-light overnight in a laminar flow hood. Previous studies have shown that after this method of sterilization, MS and loaded bio-factors remained stable and bio-active (Chen et al 1986, Li et al 2000, Schaar et al 2010, Memanishvili et al 2016).

\subsection{Electron microscopy and size distribution}

Freeze-dried PEA 4F4 MS were mounted on a specimen stub covered with adhesive carbon. Carbon tabs were sputter-coated with chromium and analyzed in a JEOL JSM-7800-F scanning electron microscope (JEOL ltd, Tokyo, Japan). Secondary electrons for surface study were detected at $1.5 \mathrm{kV}$.

For transmission electron microscope analyses, 4F4 MS were mixed with PolyBed ${ }^{\circledR} 812$ (Polysciences Inc) polymerized for $48 \mathrm{~h}$ in an oven at $+60{ }^{\circ} \mathrm{C}$. Specimens were sectioned using a Leica EM UC7 ultramicrotome (Leica Biosystems). The $60 \mathrm{~nm}$ thick sections were carefully mounted on Pioloform-coated copper grid 75 mesh and examined in a FEI Tecnai Biotwin $120 \mathrm{kV}$ electron microscope.

The average diameter of PEA 4F4 MS was estimated based on measurements on about $100 \mathrm{MS}$ from three different batches, using the scanning electron microscope software Smile View (version 2.0, JEOL). Average values were reported.

\subsection{Analysis of encapsulation efficiency and protein release}

Amount of encapsulated protein per unit weight of MS was determined by an extraction method. Five mg of VEGF-loaded PEA 4F4 MS were dissolved in $1 \mathrm{ml}$ chloroform by agitating using a shaker for $1.5 \mathrm{~h}$. One $\mathrm{ml}$ phosphate-buffered saline (PBS) $(\mathrm{pH}=7.4)$ solution containing $0.05 \%$ Tween 20 and $1 \%$ BSA was then added, and the mixture was vigorously shaken and kept rotating overnight (VWR Tube Rotator, speed $20 \mathrm{rpm}$ ) in order to extract total protein from the organic solution into the aqueous phase. After centrifugation at $10000 \times \mathrm{g}$ for $10 \mathrm{~min}$ at $+4{ }^{\circ} \mathrm{C}$, the aqueous phase was analyzed. Concentration of VEGF was determined by ELISA development kit (R\&D Systems) in quadruplicate for three independent experiments.

Encapsulation efficiency was expressed as percentage of measured loaded protein over the initial concentration used for production and represents the average of three different measurements. The total volumetric loading was expressed as the volume of VEGF loaded into the MS divided by the total volume of the MS.

Release profile of encapsulated protein from PEA 4F4 MS was determined by incubating $5 \mathrm{mg}$ of VEGFloaded MS in a test tube with $1 \mathrm{ml}$ of PBS $(\mathrm{pH}=7.4)$ solution containing $0.05 \%$ Tween 20 and $1 \%$ BSA at $+37{ }^{\circ} \mathrm{C}$. At defined time intervals $(\mathrm{t}=1,2,3, \ldots$ $90 \mathrm{~d}$ ), samples were centrifuged at $9000 \times \mathrm{g}$ for $5 \mathrm{~min}$, and medium was removed and replaced with $1 \mathrm{ml}$ fresh buffer. Concentration of VEGF was determined by ELISA development kit (R\&D Systems) in quadruplicate. Release profiles were expressed in terms of daily release $\left(\mathrm{ng} \mathrm{ml}^{-1}\right)$, and cumulative release (\% of total encapsulated protein obtained in encapsulation efficiency determination) and were plotted versus time.

After $90 \mathrm{~d}$ of incubation in buffer, VEGF-loaded MS were analyzed using a scanning electron microscope for surface monitoring.

\subsection{Animals and experimental design}

Thirty-one male, 8 weeks old, C57BL/6 mice (body weight 25-27 g, Charles River, Germany) were used. Mice were subjected to distal middle cerebral artery occlusion (dMCAO) $(\mathrm{n}=25)$, or sham procedure $(\mathrm{n}=6)$. Seven days after surgery, all shamtreated animals received PBS injection and dMCAOsubjected mice were injected with either PBS $(n=7)$, non-loaded MS $(n=9)$ or VEGF-loaded MS $(n=9)$ into cerebral cortex ipsilateral to dMCAO or sham procedure. Animals were kept in $12 \mathrm{~h}$ light/12 h dark cycles with ad libitum access to food and water. All procedures were conducted in accordance with guidelines set by the Malmö-Lund Ethical Committee for the use of laboratory animals and the European Union directive on the subject of animal rights. 


\subsection{Distal middle cerebral artery occlusion}

Animals were anesthetized with isoflurane $(3.0 \%$ induction, $1.5 \%$ maintenance) mixed with air, and were injected locally with marcaine for pain relief (20 $\mu \mathrm{l} \mathrm{of} 2.5 \mathrm{mg} \mathrm{ml}^{-1}$ stock solution, Astra Zeneca). During anesthesia and in the early recovery period $(1 \mathrm{~h})$, animals were placed on a heating pad to maintain $+37{ }^{\circ} \mathrm{C}$ body temperature. Permanent occlusion of the distal branch of the right middle cerebral artery was performed as described previously (Chen et al 1986). Briefly, after shaving the skin, an incision was made between the right eye and ear. Muscles covering the cranium were cut and opened, and a small hole was then drilled in the cranium at the level of the distal portion of the right middle cerebral artery. Dura mater was removed, and the artery was visualized and occluded by cauterization. The artery was then cut off to make sure that there was no remaining blood flow to the corresponding cortical region. After skin had been sutured, mice were injected with $1.5 \mathrm{ml}$ Ringer's solution intraperitoneally, returned to their cages, and put on a heating pad. In sham-operated mice, the distal portion of the middle cerebral artery was exposed but not occluded. Animals were allocated randomly to stroke or sham surgery and to the different experimental groups.

\subsection{MS injection}

Seven days after dMCAO, sterilized PEA 4F4 MS were resuspended in PBS to a final concentration of $10 \mu \mathrm{g} \mu \mathrm{l}^{-1}$. One $\mu \mathrm{l}$ of PBS with or without nonloaded or VEGF-loaded MS was then injected stereotaxically at a speed of $0.5 \mu \mathrm{l} \mathrm{min}{ }^{-1}$ into the cerebral cortex at the following coordinates: $1.1 \mathrm{~mm}$ anterior from bregma, $1.4 \mathrm{~mm}$ lateral from midline and $1.5 \mathrm{~mm}$ ventral from brain surface. Tooth bar was set at $-3.3 \mathrm{~mm}$. The needle was left in place for 5 min after all solution had been injected, and was then slowly removed during $1 \mathrm{~min}$. Finally, the wound was cleaned and sutured, and mice were returned to cages with heating pad.

\subsection{Behavioral tests}

During testing, evaluators were blinded for the four experimental groups: (1) sham-treated and PBSinjected ('Sham + PBS'); (2) stroke-subjected and PBS-injected ('dMCAO + PBS'); (3) stroke-subjected and non-loaded MS-injected ('dMCAO + nonloaded MS'); and (4) stroke-subjected and VEGFloaded MS-injected ('dMCAO + VEGF-loaded MS').

Modified neurological severity score ( $m N S S$ ). The mNSS represents a composite of motor (muscle status, abnormal movement), sensory (visual, tactile and proprioceptive), reflex and balance tests (Schaar et al 2010). In the version of mNSS modified for mice (Li et al 2000), the severity of impairment is defined by the score on a scale from 0 to 14 (normal score $=0$, maximum deficit score $=14)$. A composite score of 10 to 14 corresponds to severe, 5 to 9 to moderate, and 1 to 4 to mild impairment.

Adhesive removal test. To assess sensorimotor impairment, we also performed the adhesive removal test (Bouet et al 2007) as follows: Before each testing sequence, mice were placed in a transparent Perspex box $(15 \times 25 \mathrm{~cm})$ for a 1 min habituation period. Thereafter, two adhesive tapes of equal size $(0.3 \times 0.4 \mathrm{~cm})$ were applied with an equal pressure on each forepaw so that they covered the hairless part.

The order of placing the adhesive tape (right or left) was alternated between each animal and each session. Mice were then replaced in the Perspex box and the times to contact and to remove each adhesive tape were measured with a maximum of $2 \mathrm{~min}$. Mice were trained once daily for $14 \mathrm{~d}$ before $\mathrm{dMCAO}$ surgery in order to obtain optimal level of performance and limit inter-individual variation. This test was performed during $5 \mathrm{~d}$ at 1,3 , and 7 weeks after injection, and the average of the last $3 \mathrm{~d}$ of each test week was calculated.

\subsection{Immunohistochemistry}

At 8 weeks after dMCAO, animals were deeply anaesthetized with an overdose of pentobarbital and perfused transcardially with ice-cold saline followed by $4 \%$ paraformaldehyde (PFA) (Sigma-Aldrich). After post-fixation overnight (4\% PFA), brains were placed in 20\% sucrose (Sigma-Aldrich) for $24 \mathrm{~h}$. Coronal sections with $40 \mu \mathrm{m}$ thickness were cut on a freezing microtome (Leica, Germany) and kept at $-20^{\circ} \mathrm{C}$ in cryoprotective solution. Sections were preincubated in blocking solution (5\% normal donkey serum and $0.25 \%$ Triton X-100 in $0.1 \mathrm{M}$ potassiumphosphate buffer) for $1 \mathrm{~h}$ at room temperature, followed by incubation with primary antibodies overnight at $+4{ }^{\circ} \mathrm{C}$ (supplemental table S1 (available online at stacks.iop.org/BMM/15/065020/mmedia)). Fluorophore-conjugated secondary antibodies (Jackson ImmunoResearch Laboratories, Inc.) were diluted in blocking solution (1:200) and applied for 2 h. Nuclei were stained with Hoechst (Molecular Probes, ThermoFisher Scientific) for $10 \mathrm{~min}$ and sections were mounted with a medium containing DABCO as antifading reagent (Sigma Aldrich). Images were obtained using epifluorescence (BX61, Olympus) and laser scanning confocal (LSM 780, Zeiss) microscopes and a Virtual Slide Scanner (VS120-S6-W, Olympus).

\subsection{Quantifications}

All quantifications and statistical analyses were performed by researchers blinded to the experimental groups. Lesion volume was assessed in NeuNdiaminobenzidine (DAB)-stained sections $(\mathrm{n}=4)$. Single labeling for NeuN was performed with biotinylated horse anti-mouse antibody and visualized with avidin-biotin-peroxidase complex (Elite ABC 
kit; Vector Laboratories), followed by peroxidasecatalyzed $\mathrm{DAB}$ reaction. Intact areas, identified by $\mathrm{NeuN}+$ cells in the ipsilateral and contralateral hemispheres, were delineated and then measured using newCAST software (Visiopharm). Lesion area was calculated by subtracting the non-lesioned (stained) area in the injured hemisphere from the corresponding area in the contralateral hemisphere. Lesion volume was then obtained by multiplying the lesion area by the distance between the sections.

At 8 weeks after stroke, numbers of ED1+ and Iba1+ cells were counted and their morphological characterization was performed in the whole ipsilateral hemisphere in three coronal sections at $0.62,0.86$, and $1.1 \mathrm{~mm}$ anterior from bregma (selected to be the location of stroke damage and MS injection) using an epifluorescence microscope under a $40 \times$ objective. Total number of immunopositive cells was estimated stereologically using newCAST software. At least 250 cells per animal were counted in a predefined fraction of the area of interest.

Area of VEGF-immunoreactivity was determined in three images of three coronal sections at $0.62,0.86$, and $1.1 \mathrm{~mm}$ anterior from bregma by image analysis using CellSens Dimension 2010 software (Olympus). The images were selected based on the location of the stroke-induced damage and the MS injection site. Quantification was carried out in the dorsal half of the ipsilateral hemisphere. In each section, areas of immunoreactivity were identified using defined threshold for specific signal.

Using these defined parameters, the images of each region were analyzed by the software, which calculated the total area covered by pixels/specific immunopositive signal. The values corresponding to total VEGF-immunopositive areas were averaged and expressed as the percentage of area covered by VEGF per animal. The same stereological estimation was used to analyze angiogenesis expressed as vessel density by area of CD31 immunoreactivity in the injured hemisphere.

Numbers of ED1+ and Iba1+ cells, as well as their morphological characterization, were quantified in the ipsilateral hemisphere in three coronal sections at $0.62,0.86$, and $1.1 \mathrm{~mm}$ anterior from bregma using an epifluorescence microscope under a $40 \times$ objective (BX61, Olympus). We also used a previously established protocol for analysis of the degree of activation of Iba1+ microglia based on their morphological appearance (Thored et al 2009). Microglia with long and elaborated processes were classified as ramified, and considered as resting, non-activated microglia, and those with short and non-complex processes were classified as intermediate microglia. Finally, Iba1+ microglia cells without processes and somewhat rounded cell body were classified as amoeboid or rounded. They were regarded as activated microglia, expressing high levels of ED1.
Total number of immunopositive cells was estimated stereologically using newCAST software. Around 300 cells per animal were counted in a predefined fraction of the area of interest. Similarly, the total numbers of NG2+ and VEGF+/NG2+ cells were quantified stereologically in the ipsilateral and contralateral hemisphere. Colocalization of different markers was in all cases validated using a confocal microscope (LSM 780, Zeiss). For ED1 quantification, a microglia cell, stained with Iba1, was considered as positive when at least $25 \%$ of the cell body was covered by ED1 staining.

\subsection{Statistical analysis}

Statistical analysis was performed with Prism 6 Software (GraphPad), using one-way analysis of variance (ANOVA), two-way ANOVA, $\chi^{2}$ test or two-tailed unpaired t-test, with significance level at $\mathrm{P}<0.05$. Results are expressed as mean \pm standard deviation (SD).

\section{Results}

\subsection{PEA 4F4 MS can be efficiently loaded with VEGF}

Scanning electron microscopy revealed clear surface differences between VEGF-loaded and non-loaded MS. While the non-loaded spheres appeared rather smooth (figure 1(A)) with small pores on their surface (figure 1(B)), the VEGF-loaded spheres had a rougher surface (figures $1(\mathrm{C})$ and $(\mathrm{D})$ ). As shown with transmission electron microscopy, the inner ultrastructure of the VEGF-loaded spheres exhibited rounded holes (figure $1(\mathrm{E})$ ) in contrast to the non-loaded spheres, which were homogenous (figure $1(\mathrm{~F})$ ). These differences in morphology provide evidence for the presence of VEGF in the loaded MS. Accumulation of VEGF into PEA 4F4 MS was also supported by a difference in the size distribution of the particles $\left(\mathrm{P}\right.$ value $=0.0003, \chi^{2}$ test), showing higher proportion of MS with a diameter between 21 and $30 \mu \mathrm{m}$ and, conversely, lower proportion of MS with a diameter from 1 to $10 \mu \mathrm{m}$ and from 11 to $20 \mu \mathrm{m}$ among loaded as compared to non-loaded MS (figure $1(\mathrm{G})$ ).

The encapsulation efficiency of VEGF in MS, which is an important parameter to assess the usefulness of VEGF-loaded MS, was $62.7 \pm 2.5 \%$ of the initial amount of VEGF. Total volumetric loading was 0.12 .

Next, we analyzed the release profile of VEGF from loaded PEA 4F4 MS in vitro during $90 \mathrm{~d}$ by ELISA. The broad size distribution of the spheres (figure $1(G)$ ) seemed to have an impact on the release profile. The profile was characterized by a rapid initial release of $5.0 \pm 0.34 \mathrm{ng} \mathrm{d}^{-1}$ of VEGF per $\mathrm{mg}$ MS during the first $24 \mathrm{~h}$, most likely due to accelerated protein diffusion from the surface of the larger spheres. This was followed by a sustained release of 



$\mathrm{H}$



Diameter $(\mu \mathrm{m})$

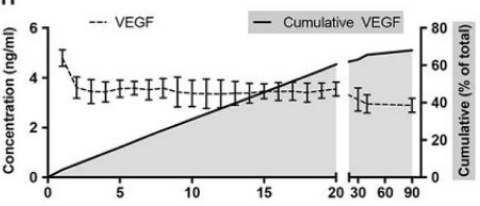

Time (days)

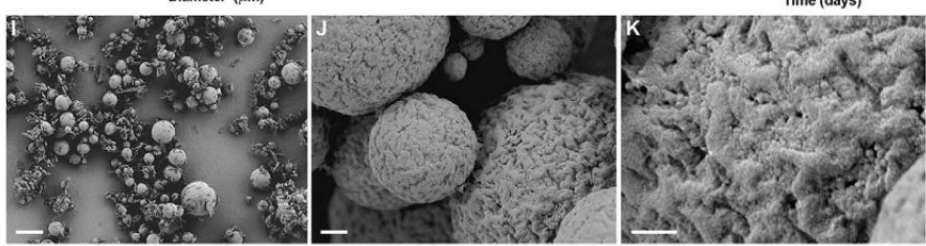

Figure 1. Morphology and VEGF release profile of PEA 4F4 MS. (A)-(D) Scanning electron micrographs at different magnifications of non-loaded (A)-(B) and VEGF-loaded (C)-(D) PEA 4F4 MS. Arrows depict small pores on the surface of non-loaded spheres. (E)-(F) Transmission electron micrographs of non-loaded (E) and VEGF-loaded (F) PEA 4F4 MS. Arrows (F) depict rounded holes in the VEGF-loaded spheres, not present in non-loaded ones (E). (G) Size distribution of non-loaded and VEGF-loaded PEA 4F4 MS (average of 100 MS from three batches for respective group). ${ }^{*} P<0.05$, Student's unpaired t-test. $(\mathrm{H})$, ELISA quantification of VEGF concentration released into the buffer from VEGF-loaded PEA 4F4 MS at different time-points, presented as daily (left axis) or cumulative release (right axis) $(n=12)$. (I)-(K) Scanning electron micrographs at different magnifications of VEGF-loaded PEA 4F4 MS after incubation in PBS containing tween and BSA for $90 \mathrm{~d}$. Scale bars $=2 \mu \mathrm{m}$ in (A) and (C), $500 \mathrm{~nm}$ in (B), (D) and (K), $1 \mu \mathrm{m}$ in (E), (F) and (J), and $20 \mu \mathrm{m}$ in (I).

$3.5 \pm 0.41 \mathrm{ng} \mathrm{d}^{-1}$ of VEGF per mg from the smaller PEA 4F4 MS (figure $1(\mathrm{H})$ ).

This second phase has been associated with polymer matrix surface erosion (Memanishvili et al 2016) in the spheres, being responsible for sustained drug diffusion to the medium during at least $90 \mathrm{~d}$. Here we found that after $90 \mathrm{~d}$ in vitro, 68\% of VEGF had been released from the PEA 4F4 MS. The amount of VEGF remaining was $30 \pm 0.4 \mathrm{ng}$ per mg MS. Partial degradation of the MS via matrix surface erosion was shown by electron microscopy (figures $1(\mathrm{I})-(\mathrm{K})$ ).

\subsection{Injection of VEGF-loaded and non-loaded PEA 4F4 MS improves functional recovery without affecting infarct volume after stroke}

Mice were subjected to $\mathrm{dMCAO}$ and, 1 week later, randomly distributed to three groups and injected with PBS, or non-loaded or VEGF-loaded MS, respectively, into cerebral cortex close to the ischemic injury. One sham-operated group was injected with PBS into cerebral cortex and used as control for stroke damage. Sensory and motor functions were evaluated before and at 2, 4 and 8 weeks after stroke using neurological score (mNSS) and adhesive removal tests. At 2 and $6 \mathrm{~d}$ after stroke or sham procedure, but before PBS or MS injection, all groups subjected to dMCAO showed significant neurological impairments compared to Sham + PBS group as evidenced by higher neurological score (figure 2(A): Interaction; $\left.\mathrm{F}_{12,108}=3.937, \mathrm{p}<0.0001\right)$. Animals injected with VEGF-loaded MS demonstrated more rapid improvement of neurological score, which did not differ from that of Sham + PBS group at 2 weeks after stroke and 1 week after injection (figure 2(A)). In contrast, stroke-subjected animals injected with PBS or non-loaded MS exhibited no signs of recovery at this time point (figure $2(\mathrm{~A})$ ). When assessed at later time-points, 4 and 8 weeks after stroke, animal groups with VEGFloaded and non-loaded MS both exhibited neurological score similar to the Sham + PBS group, whereas stroke-injured animals injected with PBS remained impaired.

We also assessed performance of the mice in the adhesive removal test. Animals subjected to dMCAO and injected with PBS needed significantly longer time to contact tape at 2 and 4 weeks and to remove tape at 2 weeks after stroke as compared to Sham + PBS group (figure 2(B): Interaction; 
A

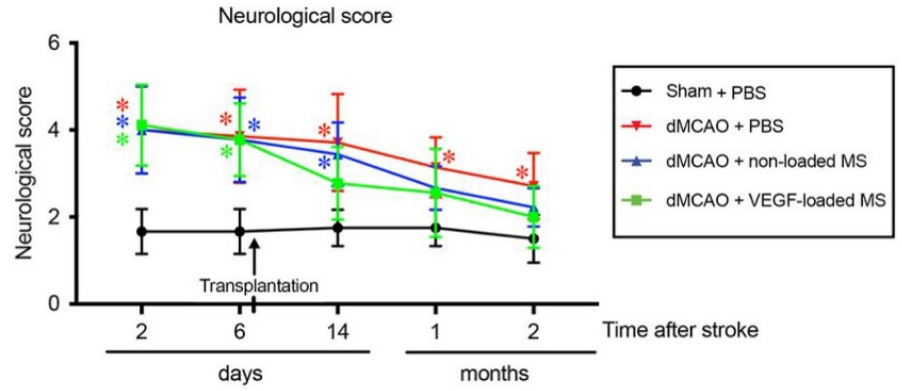

B


C


dMCAO + VEGF-loaded MS

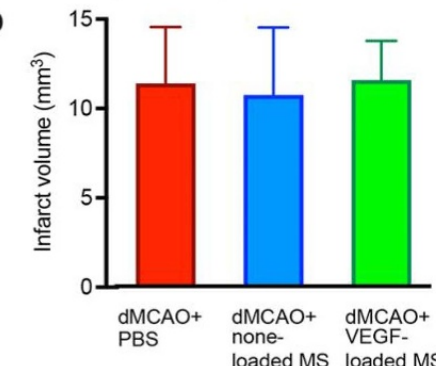

Figure 2. Effect of injection of VEGF-loaded and non-loaded PEA 4F4 MS on functional recovery and infarct volume after stroke. (A)-(B) Neurological score (A) and performance in adhesive removal test (B) at different time points after dMCAO or sham procedure and subsequent injection with PBS, or non-loaded or VEGF-loaded MS into cerebral cortex $\left(\mathrm{n}_{\text {sham }}=6\right.$; $\mathrm{n}_{\mathrm{PBS}}=7$; $\left.\mathrm{n}_{\mathrm{MS}}=9 ; \mathrm{n}_{\mathrm{VEGF}-\mathrm{MS}}=9\right) .{ }^{*} \mathrm{P}<0.05$ compared to Sham + PBS group (A) or all other groups (B), two-way ANOVA. (C)

Distribution of ischemic injury (depicted by asterisk) in mice subjected to dMCAO and injected with PBS (left), non-loaded MS (middle) or VEGF-loaded MS (right). (D) Mean volume of ischemic lesion at 8 weeks after dMCAO in animals injected with PBS, non-loaded MS and VEGF-loaded MS ( $\mathrm{n}=4$ for each group).

$\mathrm{F}_{9,81}=3.544, \mathrm{p}=0.001 ;$ and $\mathrm{F}_{9,81}=1.814, \mathrm{p}=0.07$ for time to contact and time to remove, respectively). In contrast, animals injected with either VEGFloaded or non-loaded MS showed no impairment at 2 and 4 weeks. At 8 weeks after stroke, also animals from the dMCAO + PBS group had recovered in both parameters of the adhesive removal test (figure 2(B)).

Taken together, these findings provide evidence, first, that VEGF-loaded MS can give rise to accelerated functional recovery after stroke and, second, that both implantation of loaded and non-loaded MS can promote long-term improvement. We hypothesized that the various behavioral outcomes could be correlated to the volume of the ischemic lesion but, arguing against this interpretation, found no differences between the groups (figures $2(\mathrm{C})$ and (D)).

\subsection{Injection of PEA 4F4 MS loaded with VEGF increases tissue VEGF levels and promotes angiogenesis after stroke}

We analyzed the presence of MS and distribution of VEGF at 8 weeks after stroke using anti-VEGF immunostaining (figure 3). Based on their autofluorescence, MS were detected in $78 \%$ and $56 \%$ of the animals implanted with VEGF-loaded and nonloaded MS, respectively. In those brains in which MS were not found, we observed a cavity located at the site of MS injection. It is conceivable that the 



Figure 3. Effect of injection of VEGF-loaded and non-loaded PEA 4F4 MS on VEGF immunoreactivity and angiogenesis after stroke. (A) Fluorescence photomicrographs of the coronal section at a level of injection site for PBS in sham-treated mouse cerebral cortex, showing staining with blood vessel-specific marker CD31 (red) and lack of VEGF (in green) immunoreactivity. (B)-(C) Fluorescence photomicrographs showing coronal sections of mice subjected to MCAO and injected with non-loaded (B) or VEGF-loaded (C) MS, stained for VEGF (green) and CD31 (red). (D) Fluorescence photomicrographs of the coronal section at a level of the implantation site for VEGF-loaded MS in dMCAO-injured mouse cerebral cortex, showing absence of damage in host neurons stained with MAP2 (red) around the VEGF (in green) immunoreactive MS. Insets in (A)-(D) show higher magnification of the area depicted with small white squares. (E)-(F) Quantification of area immunoreactive for VEGF (E) and of blood vessel density $(F)$ in sham-operated mice $(n=6)$ and in mice subjected to dMCAO and injected with either PBS $(n=7)$ or non-loaded $(\mathrm{n}=7)$ or VEGF-loaded MS $(\mathrm{n}=9)$ ). For each animal, $\mathrm{n}=3$ samples. ${ }^{*} P<0.05$, one-way ANOVA. Scale bar on $\mathrm{A}=200 \mu \mathrm{m}$ for the coronal sections and $30 \mu \mathrm{m}$ for large insets.

MS had been washed out during fixation, sectioning or the complex process of immunostaining on free-floating sections. Figure 3 shows a representative image of a section in which VEGF-loaded MS are clearly visible, being distributed within the parenchyma without forming clumps.

High VEGF immunoreactivity was detected close to the loaded MS, suggesting successful release of VEGF (figure 3). In agreement, quantification of the VEGF immunoreactive area in the stroke-injured hemisphere showed a significant increase of VEGF levels only in animals injected with loaded MS (figures $3(\mathrm{C})-(\mathrm{E}))$.
We hypothesized that release of VEGF from the loaded MS may increase angiogenesis, which could contribute to the accelerated functional improvement evidenced by the neurological score. Vessel density in dMCAO animals injected with PBS was similar to that of sham-operated mice and, thus, we detected no significant influence on angiogenesis by the stroke injury per se at 8 weeks. Providing evidence for the regulation of angiogenesis by VEGF released from the injected, loaded MS in stroke-subjected mice, we found markedly higher vessel density in these mice as compared to animals injected with non-loaded MS or PBS (figures 3(B) and (F)). 

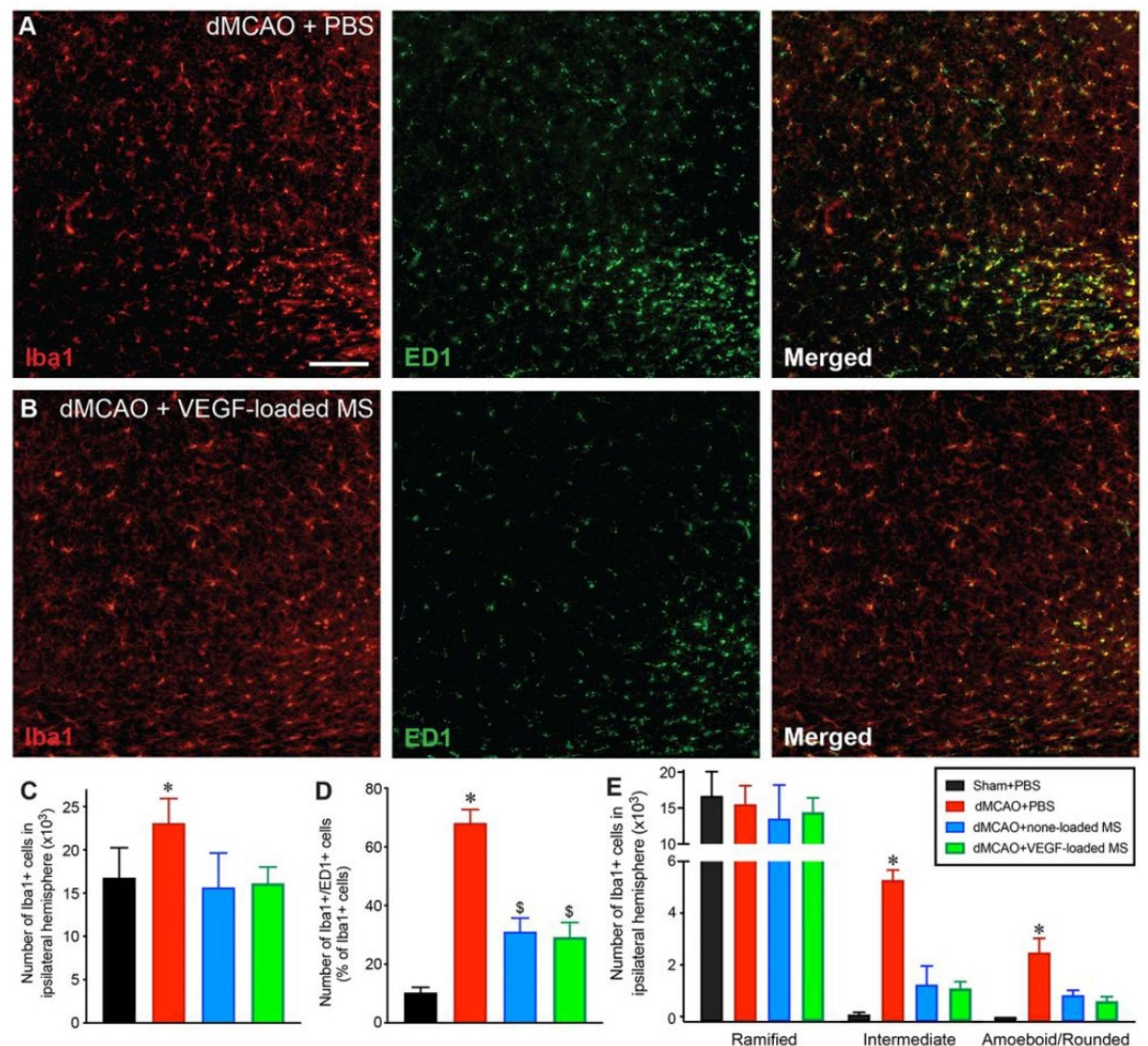

Figure 4. Effect of injection of VEGF-loaded and non-loaded PEA 4F4 MS on inflammatory response in stroke-injured brain injected with non-loaded or VEGF-loaded PEA 4F4 MS. (A)-(B) Fluorescence photomicrographs showing Iba1+ microglia (in red) and activated (ED1+) microglia (in green) in the area adjacent to the ischemic lesion of mice injected with PBS (A) or VEGF-loaded MS (B). (C)-(D) Quantification of number of Iba1+ cells (C) and percentage of Iba1+ cells expressing ED1 (D) in ipsilateral hemisphere of sham-operated mice and mice subjected to dMCAO and injected with PBS or non-loaded or VEGF-loaded MS into cerebral cortex. (E) Quantification of number of Iba1+ cells with ramified, intermediate and activated (amoeboid or rounded) morphology in ipsilateral hemisphere of the same animal groups ( $\mathrm{n}=3$ samples for each group). ${ }^{*} P<0.05$ compared with other groups, ${ }^{\$} P<0.05$ compared to sham + PBS and dMCAO + PBS groups, one-way ANOVA. Scale bar $=100 \mu \mathrm{m}$.

\subsection{Injection of both non-loaded and} VEGF-loaded PEA 4F4 MS reduces inflammatory response after stroke

Inflammation is an important player in strokeinduced neuronal loss but also contributes to tissue repair (Benakis et al 2014, Elali and Jean LeBlanc 2016, Wattananit et al 2016). To determine the effect of injected MS on the inflammatory response after stroke, the degree of microglia activation was analyzed at 8 weeks after the insult (figures $4(\mathrm{~A})$ and (B)). In animals subjected to $\mathrm{dMCAO}$ and injected with PBS, we found a significant increase both in the total number of Iba1+ cells, mainly staining resident microglia (figure $4(\mathrm{C})$ ), and the percentage of activated Iba1+/ED1+ microglia (figure $4(\mathrm{D})$ ) in the injured hemisphere as compared to sham-operated animals. We also assessed the degree of microglia activation based on their morphology. Ramified microglia are considered to be in resting state while intermediate, amoeboid and round microglia represent progressively more activated populations (Lehrmann et al 1997, Thored et al 2009). We found that the numbers of intermediate, amoeboid and round microglia were increased in $\mathrm{AMCAO}$-subjected animals injected with PBS (figure 4(E)). Interestingly, injection of both non-loaded and VEGF-loaded MS mitigated the increase of both the total number of microglia and their degree of activation after stroke (figures $4(\mathrm{~A})-(\mathrm{E}))$, with no differences between the groups.

\subsection{Injection of PEA 4F4 MS loaded with VEGF induces increased number of NG2 cells expressing VEGF after stroke}

In sham-operated and PBS-injected animals, we found VEGF immunoreactive cells in the ventricular wall including subventricular zone, and less intensely stained cells in corpus callosum. In all animal groups subjected to dMCAO, the number of VEGF + cells in the corpus callosum and the intensity of immunostaining of individual cells was substantially increased (supplemental figure S1).

Since VEGF immunoreactivity was increased in the areas surrounding the VEGF-loaded MS (figure 3 ), we determined the identity of VEGF+ cells close 
to the MS injection using co-labeling with cell typespecific antibodies. Only few GFAP+ astrocytes coexpressed VEGF in the periphery of the MS injection site (figures 5(A) and (B)). No co-localization of VEGF and the microglia- and pericyte-specific markers, Iba1 and CD13, respectively, was found (data not shown). In contrast, we found co-expression of VEGF in NG2 + cells in the corpus callosum and in the perilesion area (figure 5(C)). These cells represent a type of neuroglia distinct from mature astrocytes and oligodendrocytes as well as from microglia.

Quantification of NG2+/VEGF+ cells showed higher numbers in animals injected with MS (nonloaded or loaded with VEGF) as compared to shamoperated and stroke-subjected animals injected with PBS in both hemispheres (figure 5(D)), predominantly in the injured hemisphere. Animals injected with VEGF-loaded MS showed higher numbers of $\mathrm{NG} 2+/ \mathrm{VEGF}+$ cells as compared to those injected with non-loaded MS (figure 5(D)) in both hemispheres. In parallel, we observed increased number of $\mathrm{NG} 2+$ cells in the same groups (figure 5(E)). To what extent VEGF production was increased by the NG2+ cells themselves or VEGF originated from the MS and was taken up by the NG2+ cells could not be established.

\section{Discussion}

Here we describe, for the first time, the development of PEA MS for intracerebral injection and longterm delivery of a growth factor into the injured adult brain. The PEA MS seem particularly attractive for this purpose by combining the excellent thermal and mechanical properties of polyamides with the biocompatibility and biodegradability of polyesters (Rodriguez-Galan et al 2011, Winnacker and Rieger 2016). In addition, the PEA MS are known to show prolonged and controlled release profile of a variety of drugs and proteins, lack of side effects of degradation products, and versatility during fabrication (Winnacker and Rieger 2016). Moreover, these polymers release the drug by a combination of diffusion and polymer degradation, and because of the biodegradability do not require surgical removal when drug application is finished (Fonseca et al 2014).

Double emulsion solvent evaporation is one of the most commonly used techniques for encapsulation of hydrophilic molecules. One limitation is the moderate encapsulation efficiency (for review see (Iqbal et al 2015)). The encapsulation efficiency observed here $(62.7 \%)$ is comparable to that reported in previous studies, ranging between $51 \%$ and $60 \%$ (Yang et al Karal-Yilmaz et al 2000, Emami et al 2009, 2011, Herran et al 2013a). In these studies, the w/o/w technique was used to prepare protein-loaded polymeric microparticles, and the observed moderate encapsulation efficiency was attributed to drug leakage to the aqueous external phase.
Another important parameter in determining the kinetics of protein release from MS is the volumetric loading. Relatively high volumetric loadings are required to ensure that a large portion of the drug is released from polymers (Amsden and Cheng 1995). However, high protein loading may provoke an initial burst of fast release, as some protein becomes trapped on the surface of the polymer matrix during the manufacturing process (Huang and Brazel 2001). Low loading should also be avoided as a substantial fraction of the protein may be trapped inside the matrix, never to be released (Siegel et al 1989). Therefore, drug loadings should be kept sufficiently small to ensure that the release is governed by polymer erosion and not protein diffusion (Siegel et al 1989). Our results demonstrate that the volumetric loading of VEGF in the MS achieved here gave rise to a sustained release of the protein over time and that only a small fraction of the loaded protein was released through diffusion.

Despite the moderate encapsulation efficiency and volumetric loading in our study, the generated VEGF-loaded PEA 4F4 MS were releasing VEGF in vitro for a long time, at least up to $90 \mathrm{~d}$. This release, facilitated by the enzymatic degradation of PEA, occurs via surface erosion (Kropp et al 2014). The impregnated lipase cleaves ester bonds, triggering the degradation (Tsitlanadze et al 2004, Murase et al 2015).

It should be underlined that no accumulation of acidic byproducts is observed during the surface erosion degradation of PEAs (Mihov et al 2010, Ghaffar et al 2011, Fonseca et al 2014). As 4F4 PEA degrades, it produces small quantities of weak acidic products (Katsarava et al 2016). Thus, no drastic decrease of $\mathrm{pH}$ occurs that would provoke a significant loss of protein bioactivity. In accordance, evidence for retention of protein bioactivity using PEAs as carriers has been reported previously. VEGF phosphorylates VEGFR2 in human endothelial cells after release from PEA MS (Caolo et al 2016). Proteins such as WNT3A and BMP4, as well as fibroblast growth factor 9 (FGF9) and Chemokine (C-X-C motif) ligand 1 (CXCL1) retain their biological activity in PEAbased carriers (Said et al 2014, Caolo et al 2016, Memanishvili et al 2016). In contrast, degradation of PLGA occurs through bulk erosion and its hydrolysis leads to the accumulation of acidic monomers, lactic and glycolic acids with pKa 3.86 and 3.83, respectively, causing significant reduction of $\mathrm{pH}$ and denaturation of encapsulated proteins (Bittner et al 1998, Lai and Topp 1999, van de Weert et al 2000, Ye et al 2010). Protein instability during release has also been attributed to a local $\mathrm{pH}$ drop and the acidic microenvironment inside the microparticles (Park et al 1995, van de Weert et al 2000, Determan et al 2006, Mundargi et al 2008).

Both the non-loaded and VEGF-loaded PEA 4F4 MS enhanced behavioral recovery after injection into 



Figure 5. Effect of injection of non-loaded or VEGF-loaded PEA 4F4 MS on number of NG2+ cells expressing VEGF in stroke-injured brain. (A) Fluorescence photomicrographs showing absence of colocalization of VEGF (green) and astrocytic marker GFAP (red) in the area adjacent to the ischemic damage of a mouse injected with VEGF-loaded MS. (B) Higher magnification of the area depicted with white square in (A). (C) Fluorescence photomicrographs showing colocalization of VEGF (green) and NG2 (red) in the area adjacent to the ischemic lesion of a mouse injected with VEGF-loaded MS. (D)-(E) Quantification of number of NG2+/VEGF+ (D) and NG2 + cells (E) in ipsilateral and contralateral hemisphere of sham-operated, PBS-injected mice and mice subjected to $\mathrm{AMCAO}$ and injected with PBS, or non-loaded or VEGF-loaded MS into cerebral cortex. ${ }^{*} P<0.05$ compared to other groups, ${ }^{\$} P<0.05$ compared to sham $+\mathrm{PBS}$ and dMCAO $+\mathrm{PBS}$ groups, respectively, one-way ANOVA. Scale bars $=100 \mu \mathrm{m}$ in (A) and (C), and $20 \mu \mathrm{m}$ in (B).

the stroke-injured mouse cerebral cortex. Several lines of evidence support the conclusion that biologically active VEGF was efficiently released longterm from the loaded MS also into the brain tissue environment: First, at 7 weeks after injection (8 weeks after stroke), we observed VEGF+ PEA 4F4 MS in the parenchyma and high VEGF immunoreactivity close to the loaded PEA 4F4 MS. Accordingly, significant increase of VEGF tissue levels was found only in animals with loaded PEA 4F4 MS. Second, the PEA 4F4 MS loaded with VEGF induced a markedly higher vessel density in the injured hemisphere as compared to non-loaded PEA 4F4 MS, in agreement with the notion that VEGF is a pro-angiogenic factor
(Shibuya 2009). Third, we observed a higher number of NG2 + neuroglia cells in both hemispheres in animals with VEGF-loaded PEA 4F4 MS as compared to non-loaded MS. Interestingly, NG2+ glia cells have been implicated in vessel network formation during embryonic development (Minocha et al 2015). Finally, injection of VEGF-loaded PEA 4F4 MS gave rise to more rapid recovery (already at 2 weeks after the insult) of neurological score as compared to nonloaded PEA 4F4 MS. This specific action of VEGF is in line with previous findings demonstrating behavioral improvements following different modes of delivery of this growth factor after stroke (Greenberg and Jin 2013). 
Interestingly, intracerebral injection of not only the VEGF-loaded but also the non-loaded PEA 4F4 MS gave rise to post-stroke improvement in neurological score and adhesive removal test. It is inconceivable that these effects of the MS were due to mechanical injury to the cortical tissue, associated with inflammation, caused by the $1 \mu \mathrm{l}$ injection of the PEA 4F4 MS solution in both animal groups. Thus, intracerebral injection of PEA MS into intact brain (Memanishvili et al 2016), subconjunctival and intravitreal injection of PEA fibrils (Kropp et al 2014), intramuscular application for intradiscal delivery of PEA MS (Willems et al 2017) and intra-articular injections of PEA MS (Janssen et al 2016) evoked no significant inflammatory response. In our study, injection of stroke-damaged mice with PEA MS did not influence the size of the lesion at 8 weeks after insult. This fact suggests that the beneficial effect is not mediated through neuroprotection but by creating a microenvironment that improves tissue remodeling.

Our data provide evidence that PEA MS can exhibit direct biological effects by themselves, including improvement of post-stoke recovery, without being loaded with any factors or molecules. In line with this idea, both FGF9-loaded and non-loaded PEA supported the proliferation of fibroblasts for $5 \mathrm{~d}$ even under serum-depleted conditions (Said et al 2014). We hypothesized that the beneficial action of both non-loaded MS and loaded MS observed in our experiment could be related to an antiinflammatory effect. In support, monocytes have been reported to secrete lower levels of the proinflammatory cytokines, interleukin (IL)-6 and IL$1 \beta$, into the culture supernatant when adherent to PEA as compared to PLGA or poly(butyl methacrylate) (PBMA) (DeFife et al 2009). Also, monocytes growing on PEA exhibited more than 3-fold higher secretion of IL-1 receptor antagonist as compared to monocytes on other polymers (DeFife et al 2009). IL1 receptor antagonist is a native inhibitor of IL- $1 \beta$ that competitively binds receptors for IL- 1 and blocks pro-inflammatory signaling. We found that VEGFloaded and non-loaded PEA 4F4 MS had similar antiinflammatory effect at 7 weeks after injection into the stroke-injured brain, as evidenced by lower number of activated microglia and macrophages with intermediate and amoeboid/rounded morphology.

It has been shown that VEGF could be neuroprotective after stroke (Sun et al 2003). Our finding that the intracerebral injection of VEGF-loaded and nonloaded PEA 4F4 MS in stroke-damaged mice induced behavioral improvement but was not accompanied by alterations in infarct size argues against a neuroprotective mechanism of action. In fact, enhanced poststroke functional recovery without changes in infarct volume has been observed previously in several studies, e.g. following intracerebral transplantation of human stem cell-derived neurons and blockade of monocyte recruitment to the ischemic brain (Oki et al 2012, Tornero et al 2013, Jin et al 2014, Memanishvili et al 2016). We hypothesize that the beneficial effect of the intracerebral PEA MS injection is due to the creation of a microenvironment which improves tissue remodeling and stimulates neural plasticity.

As we and others have shown, brain inflammation including activation of microglia and intracerebral infiltration of macrophages plays an important role during the recovery phase after stroke (Rajkovic et al 2018). Taken together, our findings are consistent with the hypothesis that the recovery-promoting effect of PEA 4F4 MS could be attributed to an antiinflammatory action.

In conclusion, we demonstrate in the present study the usefulness of PEA 4F4 MS for intracerebral delivery of molecules to the injured brain. This system for long-term administration of growth factors regulating, e.g. neurogenesis, angiogenesis and neural plasticity, as shown here, and of molecules which can direct the differentiation of endogenous or transplanted stem cells to a specific cellular fate (Memanishvili et al 2016) opens up new possibilities for brain repair. The future therapeutic potential of PEA MS is strengthened by their anti-inflammatory action and biocompatibility. However, from a clinical perspective, intracerebral delivery of molecules with the aim to promote functional restoration using PEA 4F4 MS as vehicle is still in an early phase and the usefulness of this strategy needs to be explored in much more detail in experimental studies.

\section{Acknowledgments}

Lund University Bioimaging Center (LBIC) is gratefully acknowledged for providing experimental resources. We thank Professor Ramaz Katsarava, Agricultural University of Georgia, for providing the PEA 4F4, and Linda Jansson for technical assistance.

\section{Disclosure of potential conflicts of interest}

None.

\section{Funding}

This work was supported by grants from Shota Rustaveli National Science Foundation of Georgia, Swedish Research Council, Swedish Brain Foundation, Torsten Söderberg Foundation, Region Skåne, Sparbanksstiftelsen Färs \& Frosta, and Swedish Government Initiative for Strategic Research Areas (StemTherapy).

\section{ORCID iDs}

Zaal Kokaia (ㄱ) https://orcid.org/0000-0003-22962449 
Daniel Tornero (1) https://orcid.org/0000-00024812-4091

\section{References}

Amsden B and Cheng Y 1995 A generic protein delivery system based on osmotically rupturable monoliths $J$. Control. Release 33 99-105

Andres-Guerrero V et al 2015 Novel biodegradable polyesteramide microspheres for controlled drug delivery in Ophthalmology J. Control. Release 211 105-17

Benakis C, Garcia-Bonilla L, Iadecola C and Anrather J 2014 The role of microglia and myeloid immune cells in acute cerebral ischemia Front. Cell Neurosci. 8461

Bible E, Qutachi O, Chau D Y, Alexander M R, Shakesheff K M and Modo M 2012 Neo-vascularization of the stroke cavity by implantation of human neural stem cells on VEGF-releasing PLGA microparticles Biomaterials 33 $7435-46$

Bittner B, Ronneberger B, Zange R, Volland C, Anderson J M and Kissel T 1998 Bovine serum albumin loaded poly(lactide-co-glycolide) microspheres: the influence of polymer purity on particle characteristics J. Microencapsul. 15 495-514

Bouet V, Freret T, Toutain J, Divoux D, Boulouard M and Schumann-Bard P 2007 Sensorimotor and cognitive deficits after transient middle cerebral artery occlusion in the mouse Exp. Neurol. 203 555-67

Caolo V et al 2016 CXCL1 microspheres: a novel tool to stimulate arteriogenesis Drug Deliv. 23 2919-26

Chen S T, Hsu C Y, Hogan E L, Maricq H and Balentine J D 1986 A model of focal ischemic stroke in the rat: reproducible extensive cortical infarction Stroke 17 738-43

Chu C C 2012 Novel biodegradable functional amino acid-based poly(ester amide) biomaterials: design, synthesis, property and biomedical applications J. Fiber Bioeng. Inf. 1 1-31

Dailey L A, Jekel N, Fink L, Gessler T, Schmehl T, Wittmar M, Kissel T and Seeger W 2006 Investigation of the proinflammatory potential of biodegradable nanoparticle drug delivery systems in the lung Toxicol. Appl. Pharmacol. $215100-8$

DeFife K M, Grako K, Cruz-Aranda G, Price S, Chantung R, Macpherson K, Khoshabeh R, Gopalan S and Turnell W G 2009 Poly(ester amide) co-polymers promote blood and tissue compatibility J. Biomater. Sci. Polym. Ed. 20 1495-511

Determan A S, Wilson J H, Kipper M J, Wannemuehler M J and Narasimhan B 2006 Protein stability in the presence of polymer degradation products: consequences for controlled release formulations Biomaterials 27 3312-20

Di Carlo A 2009 Human and economic burden of stroke Age Ageing 38 4-5

Elali A and Jean LeBlanc N 2016 The role of monocytes in ischemic stroke pathobiology: new avenues to explore Front. Aging Neurosci. 829

Elliott Donaghue I, Tam R, Sefton M V and Shoichet M S 2014 Cell and biomolecule delivery for tissue repair and regeneration in the central nervous system J. Control. Release 190 219-27

Emami J, Hamishehkar H, Najafabadi A R, Gilani K, Minaiyan M, Mahdavi H and Nokhodchi A 2009 A novel approach to prepare insulin-loaded poly(lactic-co-glycolic acid) microcapsules and the protein stability study J. Pharm. Sci. 98 1712-31

Emerich D F, Silva E, Ali O, Mooney D, Bell W, Yu S J, Kaneko Y and Borlongan C 2010 Injectable VEGF hydrogels produce near complete neurological and anatomical protection following cerebral ischemia in rats Cell Transplant. 19 1063-71

Feigin V L et al 2014 Global and regional burden of stroke during 1990-2010: findings from the global burden of disease study 2010 Lancet $383245-54$
Ferreira L S, Gerecht S, Fuller J, Shieh H F, Vunjak-Novakovic G and Langer R 2007 Bioactive hydrogel scaffolds for controllable vascular differentiation of human embryonic stem cells Biomaterials 28 2706-17

Fonseca C, Gil M H and Simões P N 2014 Biodegradable poly(ester amide)s-a remarkableopportunity for the biomedical area: review on the synthesis,characterization and applications Prog. Polym. Sci. 39 1291-311

Ghaffar A, Draaisma G J, Mihov G, Dias A A, Schoenmakers P J and van der Wal S 2011 Monitoring the in vitro enzyme-mediated degradation of degradable poly(ester amide) for controlled drug delivery by LC-ToF-MS Biomacromolecules 12 3243-51

Greenberg D A and Jin K 2013 Vascular endothelial growth factors (VEGFs) and stroke Cell. Mol. Life Sci. 70 1753-61

Hacke W et al 2008 Thrombolysis with alteplase 3 to 4.5 hours after acute ischemic stroke N. Engl. J. Med. 359 1317-29

Hayashi T, Abe K and Itoyama Y 1998 Reduction of ischemic damage by application of vascular endothelial growth factor in rat brain after transient ischemia J. Cereb. Blood Flow Metab. 18 887-95

Hemmrich K, Salber J, Meersch M, Wiesemann U, Gries T, Pallua $\mathrm{N}$ and Klee D 2008 Three-dimensional nonwoven scaffolds from a novel biodegradable poly(ester amide) for tissue engineering applications J. Mater. Sci. Mater. Med. 19 257-67

Herran E, Perez-Gonzalez R, Igartua M, Pedraz J L, Carro E and Hernandez R M 2013a VEGF-releasing biodegradable nanospheres administered by craniotomy: a novel therapeutic approach in the APP/Ps1 mouse model of Alzheimer's disease J. Control. Release 170 111-9

Herran E, Ruiz-Ortega J A, Aristieta A, Igartua M, Requejo C, Lafuente J V, Ugedo L, Pedraz J L and Hernandez R M 2013b In vivo administration of VEGF- and GDNF-releasing biodegradable polymeric microspheres in a severe lesion model of Parkinson's disease Eur. J. Pharm. Biopharm. 85 $1183-90$

Huang X and Brazel C S 2001 On the importance and mechanisms of burst release in matrix-controlled drug delivery systems J. Control. Release 73 121-36

Huang Y, Wang L, Li S, Liu X, Lee K, Verbeken E, van de Werf F and de Scheerder I 2006 Stent-based tempamine delivery on neointimal formation in a porcine coronary model Acute Card. Care 8 210-6

Iqbal M, Zafar N, Fessi H and Elaissari A 2015 Double emulsion solvent evaporation techniques used for drug encapsulation Int. J. Pharm. 496 173-90

Janssen M, Timur U T, Woike N, Welting T J, Draaisma G, Giibels M, van Rhijn L W, Mihov G, Thies J and Emans P J 2016 Celecoxib-loaded PEA microspheres as an auto regulatory drug-delivery system after intra-articular injection $J$. Control. Release 244 30-40

Jikia D, Chkhaidze N, Imedashvili E, Mgaloblishvili I, Tsitlanadze G, Katsarava R, Glenn Morris J Jr and Sulakvelidze A 2005 The use of a novel biodegradable preparation capable of the sustained release of bacteriophages and ciprofloxacin, in the complex treatment of multidrug-resistant Staphylococcus aureus-infected local radiation injuries caused by exposure to Sr90 Clin. Exp. Dermatol. 30 23-26

Jin Q, Cheng J, Liu Y, Wu J, Wang X, Wei S, Zhou X, Qin Z, Jia J and Zhen X 2014 Improvement of functional recovery by chronic metformin treatment is associated with enhanced alternative activation of microglia/macrophages and increased angiogenesis and neurogenesis following experimental stroke Brain Behav. Immun. 40 131-42

Kaplan O, Zarubova J, Mikulova B, Filova E, Bartova J, Bacakova L and Brynda E 2016 Enhanced mitogenic activity of recombinant human vascular endothelial growth factor VEGF121 expressed in E. coli origami B (DE3) with molecular chaperones PLoS One 11 e0163697

Karal-Yilmaz O, Serhatli M, Baysal K and Baysal B M 2011 Preparation and in vitro characterization of vascular endothelial growth factor (VEGF)-loaded 
poly(D,L-lactic-co-glycolic acid) microspheres using a double emulsion/solvent evaporation technique $J$. Microencapsul. 28 46-54

Katsarava R, Kulikova N and Puiggalí J 2016 Biodegradable polymers composed of amino acid based

diamine-diesters-promising materials for the applications in regenerative medicine J.J. Regener. Med. 1012

Knight D K, Gillies E R and Mequanint K 2014 Biomimetic L-aspartic acid-derived functional poly(ester amide)s for vascular tissue engineering Acta Biomater. 10 3484-96

Kropp M et al 2014 Biocompatibility of poly(ester amide) (PEA) microfibrils in ocular tissues Polymers 6 243-60

Krum J M, Mani N and Rosenstein J M 2002 Angiogenic and astroglial responses to vascular endothelial growth factor administration in adult rat brain Neuroscience 110 589-604

Lai M C and Topp E M 1999 Solid-state chemical stability of proteins and peptides J. Pharm. Sci. 88 489-500

Lee E and Son H 2009 Adult hippocampal neurogenesis and related neurotrophic factors BMB Rep. 42 239-44

Lee S H et al 2002 In-vivo biocompatibility evaluation of stents coated with a new biodegradable elastomeric and functional polymer Coron. Artery Dis. 13 237-41

Lehrmann E, Christensen T, Zimmer J, Diemer N H and Finsen B 1997 Microglial and macrophage reactions mark progressive changes and define the penumbra in the rat neocortex and striatum after transient middle cerebral artery occlusion $J$. Comp. Neurol. 386 461-76

Li Y, Chopp M, Chen J, Wang L, Gautam S C, Xu Y X and Zhang Z 2000 Intrastriatal transplantation of bone marrow nonhematopoietic cells improves functional recovery after stroke in adult mice J. Cereb. Blood Flow Metab. 20 1311-9

Liu Y, Ghassemi A H, Hennink W E and Schwendeman S P 2012 The microclimate $\mathrm{pH}$ in poly(D,L-lactide-co-hydroxymethyl glycolide) microspheres during biodegradation Biomaterials 33 7584-93

Lo E H, Singhal A B, Torchilin V P and Abbott N J 2001 Drug delivery to damaged brain Brain Res. Rev. 38 140-8

London N J, Chiang A and Haller J A 2011 The dexamethasone drug delivery system: indications and evidence $A d v$. Ther. 28 351-66

Makadia H K and Siegel S J 2011 Poly lactic-co-glycolic acid (PLGA) as biodegradable controlled drug delivery carrier Polymers 3 1377-97

Markoishvili K, Tsitlanadze G, Katsarava R, Morris J G Jr and Sulakvelidze A 2002 A novel sustained-release matrix based on biodegradable poly(ester amide)s and impregnated with bacteriophages and an antibiotic shows promise in management of infected venous stasis ulcers and other poorly healing wounds Int. J. Dermatol. 41 453-8

Memanishvili T, Kupatadze N, Tugushi D, Katsarava R, Wattananit S, Hara N, Tornero D and Kokaia Z 2016 Generation of cortical neurons from human induced-pluripotent stem cells by biodegradable polymeric microspheres loaded with priming factors Biomed. Mater. 11025011

Mihov G, Draaisma G, Dias A, Turnell B and Gomurashvili Z 2010 Degradable polyesteramides: A novel platform for sustained drug delivery J. Control. Release 148 e46-e47

Minocha S, Valloton D, Brunet I, Eichmann A, Hornung J P and Lebrand C 2015 NG2 glia are required for vessel network formation during embryonic development Elife 4 e09102

Mundargi R C, Babu V R, Rangaswamy V, Patel P and Aminabhavi T M 2008 Nano/micro technologies for delivering macromolecular therapeutics using poly(D,L-lactide-co-glycolide) and its derivatives J. Control. Release 125 193-209

Murase S, Lv L-P, Kaltbeitzel A, Landfester K, Del Valle L, Katsarava R, Puiggali J and Crespy D 2015 Amino acid-based poly(ester amide) nanofibers for tailored enzymatic degradation prepared by miniemulsion-electrospinning RSC Adv. 555006

Oki K et al 2012 Human-induced pluripotent stem cells form functional neurons and improve recovery after grafting in stroke-damaged brain Stem Cells 30 1120-33
Orive G, Anitua E, Pedraz J L and Emerich D F 2009 Biomaterials for promoting brain protection, repair and regeneration Nat. Rev. Neurosci. 10 682-92

Palmer T D, Willhoite A R and Gage F H 2000 Vascular niche for adult hippocampal neurogenesis J. Comp. Neurol. 425 479-94

Pardridge W M 2005 The blood-brain barrier: bottleneck in brain drug development NeuroRx 2 3-14

Park T, Lu W and Crotts G 1995 Importance of in vitro experimental conditions on protein release kinetics, stability and polymer degradation in protein encapsulated poly (D,L-lactic acid-co glycolic acid) microspheres J. Control. Release 33 211-22

Quittet M S et al 2015 Effects of mesenchymal stem cell therapy, in association with pharmacologically active microcarriers releasing VEGF, in an ischaemic stroke model in the rat Acta Biomater. 15 77-88

Rajkovic O, Potjewyd G and Pinteaux E 2018 Regenerative medicine therapies for targeting neuroinflammation after stroke Front. Neurol. 9734

Rodriguez-Galan A, Franco L and Puiggali J 2011 Degradable poly(ester amide)s for biomedical applications Polymers 3 65-99

Rosenstein J M, Mani N, Silverman W F and Krum J M 1998 Patterns of brain angiogenesis after vascular endothelial growth factor administration in vitro and in vivo Proc. Natl Acad. Sci. USA 95 7086-91

Said S S, Pickering J G and Mequanint K 2014 Controlled delivery of fibroblast growth factor-9 from biodegradable poly(ester amide) fibers for building functional neovasculature Pharm. Res. 31 3335-47

Schaar K L, Brenneman M M and Savitz S I 2010 Functional assessments in the rodent stroke model Exp. Transl. Stroke Med. 213

Shibuya M 2009 Brain angiogenesis in developmental and pathological processes: therapeutic aspects of vascular endothelial growth factor Febs J. 276 4636-43

Siegel R, Kost J and Langer R 1989 Mechanistic studies of macromolecular drug release from macroporous polymers. I. Experiments and preliminary theory concerning completeness of drug release J. Control. Release 8 223-36

Storkebaum E, Lambrechts D and Carmeliet P 2004 VEGF: once regarded as a specific angiogenic factor, now implicated in neuroprotection Bioessays 26 943-54

Sun Y, Jin K, Xie L, Childs J, Mao X O, Logvinova A and Greenberg D A 2003 VEGF-induced neuroprotection, neurogenesis, and angiogenesis after focal cerebral ischemia J. Clin. Invest. 111 1843-51

Sundback C A, Shyu J Y, Wang Y, Faquin W C, Langer R S, Vacanti J P and Hadlock T A 2005 Biocompatibility analysis of poly(glycerol sebacate) as a nerve guide material Biomaterials 26 5454-64

Thored P et al 2009 Long-term accumulation of microglia with proneurogenic phenotype concomitant with persistent neurogenesis in adult subventricular zone after stroke Glia $57835-49$

Tornero D et al 2013 Human induced pluripotent stem cell-derived cortical neurons integrate in stroke-injured cortex and improve functional recovery Brain 136 3561-77

Tsitlanadze G, Kviria T, Katsarava R and Chu C C 2004 In vitro enzymatic biodegradation of amino acid based poly(ester amide)s biomaterials J. Mater. Sci. Mater. Med. 15 185-90

van de Weert M, Hennink W E and Jiskoot W 2000 Protein instability in poly(lactic-co-glycolic acid) microparticles Pharm. Res. 17 1159-67

Wattananit S et al 2016 Monocyte-derived macrophages contribute to spontaneous long-term functional recovery after stroke in mice J. Neurosci. 36 4182-95

Willems N et al 2017 Safety of intradiscal injection and biocompatibility of polyester amide microspheres in a canine model predisposed to intervertebral disc degeneration J. Biomed. Mater. Res. B 105 707-14 
Winnacker M and Rieger B 2016 Poly(ester amide)s: recent insights into synthesis, stability and biomedical applications Polym. Chem. 7 7039-46

Wittko I M, Schanzer A, Kuzmichev A, Schneider F T, Shibuya M, Raab S and Plate K H 2009 VEGFR-1 regulates adult olfactory bulb neurogenesis and migration of neural progenitors in the rostral migratory stream in vivo J. Neurosci. 29 8704-14

Yang -Y-Y, Chung T-S, Bai X-L and Chan W-K 2000 Effect of preparation conditions on morphology and release profiles of biodegradable polymeric microspheres containing protein fabricated by double-emulsion method Chem. Eng. Sci. 55 2223-36
Ye M, Kim S and Park K 2010 Issues in long-term protein delivery using biodegradable microparticles J. Control. Release 146 241-60

Zhang H T, Scott P A, Morbidelli L, Peak S, Moore J, Turley H, Harris A L, Ziche M and Bicknell R 2000a The 121 amino acid isoform of vascular endothelial growth factor is more strongly tumorigenic than other splice variants in vivo $\mathrm{Br}$. J. Cancer 83 63-68

Zhang Z G, Zhang L, Jiang Q, Zhang R, Davies K, Powers C, Bruggen $\mathrm{N}$ and Chopp $\mathrm{M}$ 2000b VEGF enhances angiogenesis and promotes blood-brain barrier leakage in the ischemic brain J. Clin. Invest. 106 829-38

Zhuang J, Fang R H and Zhang L 2017 Preparation of particulate polymeric therapeutics for medical applications Small Methods 11700147 